\title{
Mucormycosis of Paranasal Sinuses of Odontogenic Origin Post COVID19 Infection: A Case Series
}

\author{
Poonam Kumar Saidha $^{1} \cdot$ Sahil Kapoor $^{1} \cdot$ Pooja Das $^{1}$ (i) $\cdot$ Ayushi Gupta $^{1} \cdot$ \\ Vikas Kakkar $^{1} \cdot$ Arun Kumar $^{1} \cdot$ Varun Arya $^{1}$
}

Received: 13 April 2021 / Accepted: 16 May 2021/Published online: 17 June 2021

(C) Association of Otolaryngologists of India 2021

\begin{abstract}
The Severe acute respiratory syndrome and the Middle East respiratory syndromes emerged in 2002 and 2012 respectively. Currently the world is witnessing a global pandemic caused by a novel coronavirus, the severe acute respiratory syndrome coronavirus 2 (SARS CoV- 2) causing the Coronavirus 2019 (COVID-19). Mucormycosis is a fungal infection primarily affecting individuals with an immunocompromised state like diabetes mellitus, malignancies etc. Patients who have or have had COVID-19 infection with pre-existing uncontrolled Type 2 Diabetes mellitus are presumably more vulnerable for emergence of fungal infections cases. This article presents a report of 6 cases with histopathological proven mucormycosis associated with COVID-19 and uncontrolled Diabetes mellitus.
\end{abstract}

Keywords Mucormycosis - Type 2 diabetes mellitus . Immunocompromised · COVID-19 - Amphotericin B

\section{Introduction}

Mucormycosis is a severe opportunistic fungal infection caused by fungi belonging to the family called Mucorales of which Rhizopus species being the most common causative pathogen. The infection occurs mainly in immunocompromised patients, especially in individuals diagnosed with uncontrolled diabetes mellitus or haematological malignancies [1-3]. It is recognised as one of the most rapidly progressive morbid form of fungal infection in

Pooja Das

drpooja.2610@gmail.com

1 Department of ENT at SGT Hospital, SGT University, C.V Raman Hostel, Gurgaon, India humans with a high mortality [4]. Patients with severe acute respiratory syndrome coronavirus 2 (SARS-CoV-2) infection developing coronavirus disease (COVID-19), which can be associated with signifcant and sustained lymphopenia thereby compromising the immune system [5-7]. The correlation between COVID-19 and Mucormycosis though unexpected but is not at all surprising. Because the major risk factor for Mucormycosis is down-regulation of immune system specifically in uncontrolled diabetes mellitus which is also considered to be a high-risk factor for COVID-19. Secondly, steroids-which are known to suppress immunity also form the line of treatment which has shown to decrease COVID-19 mortality. Henceforth it is the combination of COVID-19, with decreased immunity and the steroids which probably predispose a patient to Mucormycosis.

We present a report of six cases who presented to the Department of ENT with paranasal mucormycosis associated with past Covid-19 infection.

\section{Study Design- Clinical Research}

Study Setting-This is a hospital based study conducted in the Department of ENT at SGT Medical College Hospital and Research Institute, Gurugram.

\section{Case Presentation}

\section{Case 1}

A 39 years male patient presented with complaints of pus discharging from gums and loose teeth of left upper jaw associated with swelling of left cheek and facial pain since 
3 months. On intra oral examination multiple pus draining sinuses were observed in left upper gingiva (Fig. 1). NCCT PNS showed complete obliteration of left maxillary sinus with partially obliterated right maxillary and anterior ethmoidal sinuses with a hyper dense mass and disintegration of bone trabeculae of maxilla (Fig. 2). Hematological investigations revealed elevated ESR, C-Reactive Protein and raised fasting blood sugar (FBS) level to $180 \mathrm{mg} / \mathrm{dl}$ and $\mathrm{HbA} 1 \mathrm{c}$ of $9.5 \%$. KOH mount and fungal culture of pus was sent which revealed growth of Aseptate hyphae with branched sporangiospores, bearing spherical sporangia at the tip suggestive of Mucor. RT-PCR for COVID 19 showed a negative result although Covid-19 antibody titre revealed count of $173 \mathrm{AU} / \mathrm{ml}$ indicating previous exposure to Covid-19 virus. Pre-operatively patient was prescribed with Tab. Iatroconazole $200 \mathrm{mg}$ twice daily. He was then posted for FESS with surgical debridement of left and right maxillary sinus. Postoperative period remained uneventful, topical douching of both nasal cavities and antrum with Amphotericin B solution was done twice daily, Inj Liposomal Amphotericin B was started at a dose of $1 \mathrm{mg} / \mathrm{kg}$ body weight/day to a cumulative dose of $2 \mathrm{~g}$ along with oral hypoglycemic agents. Routine endoscopic examination revealed healthy mucosa of maxillary sinus (Fig. 3).

\section{Case 2}

A 29 years male patient reported with complaints of dull aching facial pain with swelling over right maxilla and numbness of right side of upper lip since 1 month. He complained of headache since 20 days. History taking revealed she was infected with COVID-19 infection 2 months earlier, although RT-PCR report was found to be negative on admission. Local examination revealed diffuse swelling and tenderness over right side of face along with tenderness in bilateral ethmoid region (Fig. 4). He was a

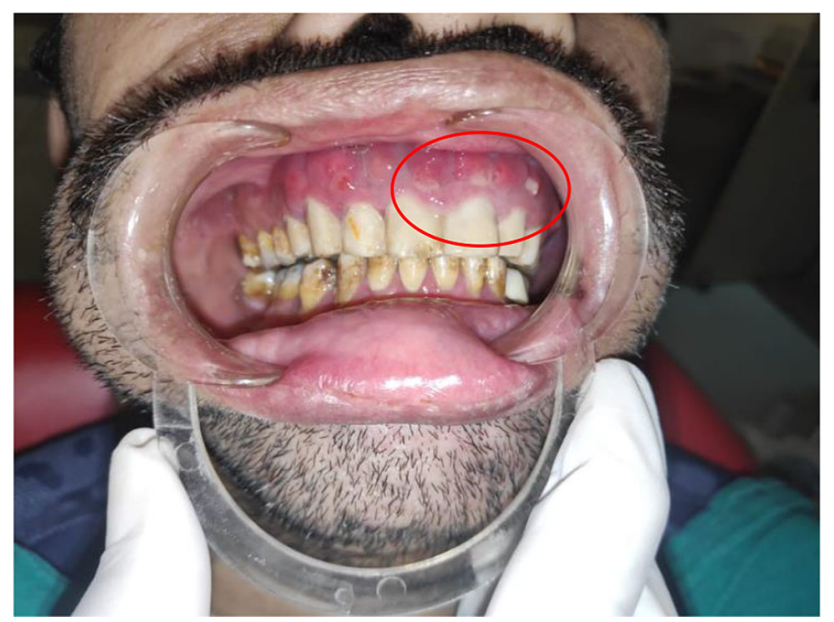

Fig. 1 Showing multiple draining sinuses in left upper gingiva

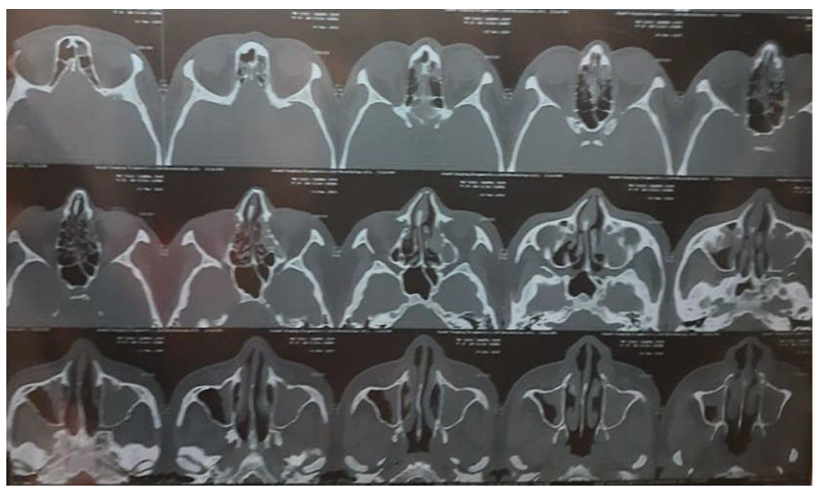

Fig. 2 NCCT PNS depicting complete obliteration of left and partial obliteration of right maxillary sinus

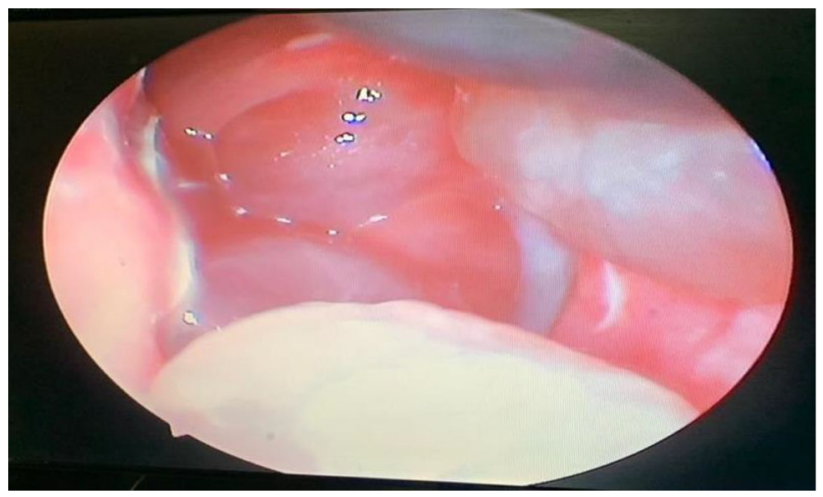

Fig. 3 Endoscopic view showing postoperative normal mucosa of maxillary sinus

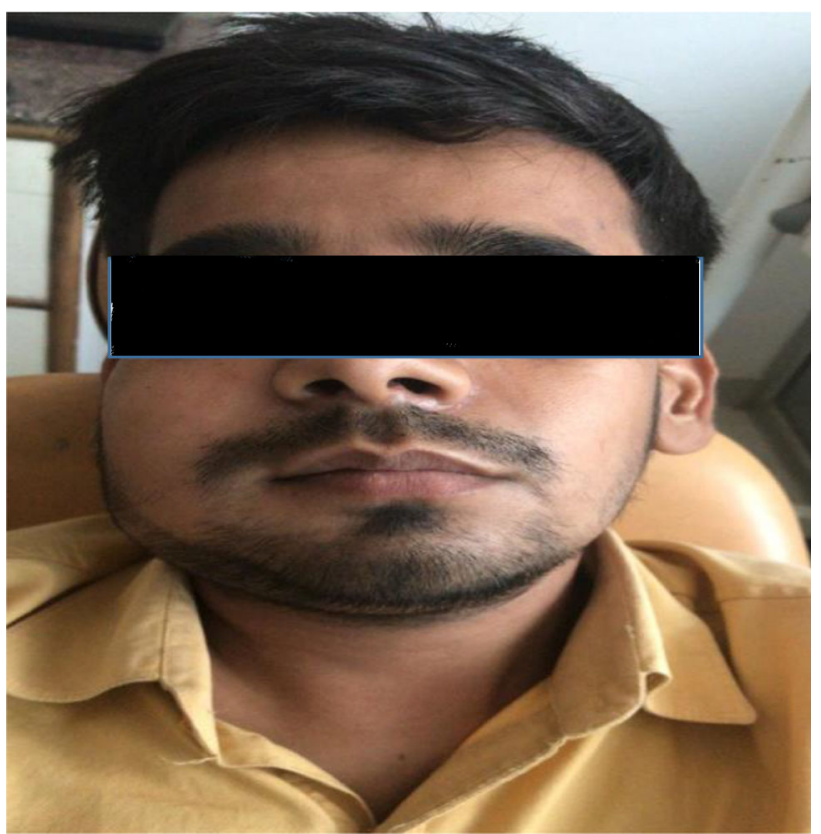

Fig. 4 Showing swelling in right maxilla 
diagnosed with Diabetes mellitus on admission. Laboratory investigations revealed raised ESR and TLC with raised random blood sugar level to $257 \mathrm{mg} / \mathrm{dl}$. Radiological imaging illustrated obliteration of right maxillary sinus with a hyper dense mass involving the anterior ethmoidal sinuses on both sides. Nasal and maxillary antrum swab was taken and sent for culture in Sabouraud's dextrose agar media and KOH mount which showed growth of broad aseptate hyphae with sporangium suggestive of mucor. Patient was posted for FESS and tissue from maxillary sinus was sent for histopathology which further confirmed diagnosis of mucormycosis on detection of aseptate hyphae (Fig. 5). Post operatively nasal and antral douching with Amphotericin B was started with Oral Posaconazole for 14 days. Diabetes was managed with insulin adjusted as per sliding scale.

\section{Case 3}

A 56 years male presented with facial pain and foul odour from mouth. He had history of Covid-19 three months back and is a known case of type 2 Diabetes mellitus. Intra oral examination revealed mobile teeth with blackish discolouration of upper teeth sockets and bone erosion over hard palate. NCCT face and PNS showed obliteration of both maxillary sinuses with a hyper dense mass involving the floor of hard palate. Laboratory parameters showed elevated fasting blood sugar levels to $240 \mathrm{mg} / \mathrm{dl}$. Swab collected and send for $\mathrm{KOH}$ mount and culture in Sabouraud's dextrose agar medium which illustrated growth of aseptate hyphae identified as mucor species. Patient was advised hospital admission for further management however she left against medical advice due to financial constraints.

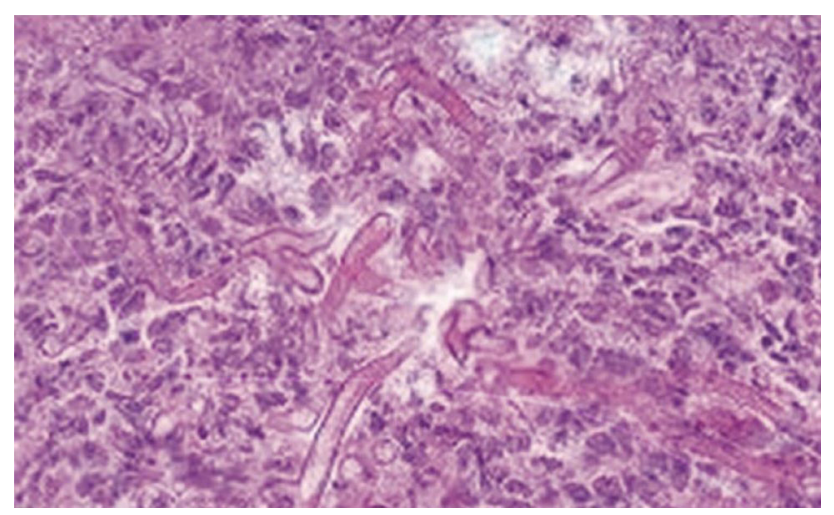

Fig. 5 Histopathology of maxillary sinus showing broad, aeptate hyphae and branching at right angles resembling Mucor

\section{Case 4}

A 68 years female reported with complaints of sudden onset of unconsciousness, throat pain and poor oral intake. Patient was referred from a local hospital where diagnosis of Diabetic ketoacidosis associated with type 2 Diabetes mellitus, hypertension, hypoalbuminemia, sepsis associated with mucormycosis and chronic kidney disease was made. FBS was $390 \mathrm{mg} / \mathrm{dl}$ on presentation. Patient had foul breath and a sinus was noticeable over right upper jaw which showed blackish discharge arising from right maxillary sinus. She was admitted to ICU and intubated. Covid19 antibody (IgG) titre was $186 \mathrm{AU} / \mathrm{ml}$ indicating previous exposure of patient to viral pathogen. Patients condition deteriorated and she expired the next day of intubation.

\section{Case 5}

A 35 years male patient presented with complaints of foul smelling nasal discharge which was blood tinged occasionally, headache and facial pain since one month. He complained of loss of upper right premolar tooth on its own for which he consulted a dentist and further intra oral examination revealed necrotic alveolar bone with evidence of foul discharge and tenderness in relation to right maxillary edentulous region. Radiographic examination showed opacification of right maxillary sinus. NCCT PNS Scan revealed non homogenous opacification of right maxillary sinus causing destruction of right osteo-meatal unit extending to middle meatus, and ethmoid sinuses causing destruction of walls of maxillary and ethmoid sinuses. Haematological parameters revealed raised ESR and TLC with a rise in random blood sugar level of $260 \mathrm{mg} / \mathrm{dl}$. Patient gave history of hospital admission three months back due to respiratory distress where he was diagnosed with Covid-19 infection and was under treatment of IV Steroids $1 \mathrm{mg} / \mathrm{kg}$ body weight/day and Remedesvir intravenously with loading dose of $200 \mathrm{mg}$ on day 1 followed by IV maintenance doses of $100 \mathrm{mg}$ for 5 days. Patient underwent sino nasal debridement and the necrotic bone of right maxillary edentulous region was removed. Histopathological examination of biopsied specimen showed necrosed tissue with fungal hyphae which were non septate, sporangiospores with spores suggestive of mucormycosis. He was administered Amphotericin B $1 \mathrm{mg} / \mathrm{kg}$ body weight intravenously with nasal douching. Blood sugar levels were controlled with insulin. Healing was satisfactory in follow up.

\section{Case 6}

A 55 years female known diabetic for last 10 years on oral hypoglycaemic agents presented with facial pain and with 
headache associated with facial cellulitis predominant on left side of face since 20-25 days. Physical examination revealed tenderness in left maxillary and left ethmoid region. Skin over left maxillary sinus showed signs of inflammation. Haematological parameters showed raised ESR and TLC with fasting blood sugar level of $245 \mathrm{mg} / \mathrm{dl}$ and HbA1c of $8.5 \%$. Radiological imaging illustrated complete obliteration of left maxillary sinus with a hyper dense mass with erosion of medial wall of maxillary antrum involving the left anterior and posterior ethmoidal sinuses. RT-PCR test was negative for Covid-19 although antibody titre was $178 \mathrm{AU} / \mathrm{ml}$ indicating a previous exposure. Patient was posted for FESS, postoperative period remained uneventful. Biopsied tissue was sent for histopathology which showed growth of aseptate hyphae with sporangium suggestive of mucormycosis. Patient was under cover of Inj amphotericin B and topical amphotericin solution for nasal douching. Follow up visits showed good prognosis.

\section{Discussion}

Mucormycosis is the third invasive mycosis in order of importance after candidiasis and aspergillosis and is caused by fungi of the class Zygomycetes. The most important species in order of frequency is Rhizopus arrhizus [8]. The rhino-maxillary form of the disease, a subdivision of the rhino-cerebral form, begins with the inhalation of the fungus by a susceptible individual [9]. Once the spores have penetrated the lungs or subcutaneous tissues, they are met by the first line of defence, mononuclear and polynuclear phagocytes [8]. The phagocytes of the healthy host are able to kill the spores of Mucorales by generating oxidative metabolites [8]. Uncontrolled diabetes mellitus, because of ketoacidosis, can alter the normal immunologic response of patients to infections [10]. Such patients have decreased granulocyte phagocytic ability with altered polymorphonuclear leukocyte response [10]. Fungal invasion of oro-nasal cavity or paranasal sinuses of susceptible host causes consistent symptoms, sinusitis or periorbital cellulitis, and facial numbness, followed by the onset of conjunctival suffusion, blurry vision, and soft tissue swelling followed by eschar formation and necrosis of nasofacial region [11-12]. A clinical suspicion of mucormycosis requires confirmation by histopathological examination preferably a CT scan of the maxilla and orbit, showing membrane or periosteal thickening and bony disruption [13].

COVID-19 infection results in dysregulation of immune system with reduction of $\mathrm{T}$ lymphocytes, $\mathrm{CD} 4+\mathrm{T}$ and CD8 $+\mathrm{T}$ cells. A multifactorial picture of pre-existing Diabetes mellitus and systemic immune changes due to
COVID-19 infection which further leading to secondary infections thereby increasing mortality and morbidity [14]. Studies show that SARS-CoV and SARS-CoV 2 are from the same species and have similar biological characteristics and prevalence [15]. Incidence of fungal infection in patients with SARS (2003) was 14.8-27\% and even higher in severely ill ones upto $21.9-33 \%$ [16-17]. In all cases of death, fungal infection was a major cause for SARS patients accounting for 25-73.7\% [18, 19]. In all cases presented in this article, an association of Mucormycosis with poorly controlled diabetes mellitus and COVID-19 cannot be overlooked. The global mucormycosis case fatality rate is $46 \%$. Rapid diagnosis at an early stage with surgical debridement and anti-fungal treatment enables speedy recovery. Henceforth a clinical suspicion requires confirmation by histopathological examination. Liposomal amphotericin B is the drug of choice, other anti-fungal agents used are Posaconazole and Itraconazole. Prognosis is favourable when surgical and anti-fungal therapy are combined with efficient blood sugar control.

\section{Conclusion}

Co-existence of bacterial and fungal infections forming a triad with COVID-19 patients has been seen. Covid-19 patients have showed over expression of inflammatory cytokines and impaired cell mediated immune response with decreased CD4 $+\mathrm{T}$ and CD8 $+\mathrm{T}$ cell counts thus indicating high susceptibility to fungal co infection.

Use of broad spectrum antibiotics along with steroids which act as the main line of therapy for Covid-19 infection to reduce mortality acts as an exacerbating factor to further supress the immune response. Diabetes mellitus is another important risk factor and the presence of both have additional effects in causing secondary fungal infections. Foul smelling nasal discharge with headache and foul breath in a diabetic and COVID-19 patient should be considered highly suspicious of mucormycosis. Early diagnosis with efficient treatment along with surgical debridement can enhance recovery. Prognosis may improve with rapid diagnosis, prompt management and reversibility of underlying risk factors can enhance recovery and survival rates to a larger extent.

\section{References}

1. Bassetti M, Bouza E (2017) Invasive mould infections in the ICU setting: complexities and solutions. J Antimicrob Chemother 72:i39-47

2. Lin E, Moua T, Limper AH (2017) Pulmonary mucormycosis: clinical features and outcomes. Infection 45:443-448 
3. Peng M, Meng H, Sun Y et al (2019) Clinical features of pulmonary mucormycosis in patients with diferent immune status. J Thorac Dis 11:5042-5052

4. Jagdish C, Reetika S, Pallavi S, Jayanthi S, Divya P, Balasubramanya A (2012) Mucormycosis of the paranasal sinus with gas forming maxillary osteomyelitis-a case report. Internet J Microbiol, vol.10, no.1

5. Liu J, Li S, Liu J et al (2020) Longitudinal characteristics of lymphocyte responses and cytokine profles in the peripheral blood of SARSCoV-2 infected patients. EBioMedicine 55:102763

6. Zhu N, Zhang D, Wang W et al (2020) A novel coronavirus from patients with pneumonia in China, 2019. N Engl J Med 382:727-733

7. Lin L, Lu L, Cao W, Li T. Hypothesis for potential pathogenesis of SARS-CoV-2 infection-a review of immune changes in patients with viral pneumonia

8. Bouza E, Munoz P, Guinea J (2006) Mucormycosis: an emerging disease? Clin Microbiol Infect 12(7):7-23

9. Greenberg MS (2003) Ulcerative vesicular and bullous lesions. In: Greenberg MS, Glick M (eds) Burket's oral medicine diagnosis and treatment. Elsevier, Amsterdam

10. Brown OE, Finn R (1986) Mucormycosis of the mandible. J Oral Maxillofac Surg 44(2):132-136

11. Spellberg B, Edwards J Jr, Ibrahim A (2005) Novel perspectives on mucormycosis: pathophysiology, presentation, and management. Clin Microbiol Rev 18(3):556-569

12. Fogarty C, Regennitter F, Viozzi CF (2006) Invasive fungal infection of the Maxilla following dental extractions in a patient with chronic obstructive pulmonary disease. J Can Dent Assoc 72(2):149-152
13. Pandey A, Bansal V, Asthana AK, Trivedi V, Madan M, Das A (2011) Maxillary osteomyelitis by mucormycosis: report of four cases. Int J Infect Dis 15(1):e66-e69

14 Gangneux JP, Bougnoux ME, Dannaoui E, Cornet M, Ralph ZJ (2020) Invasive fungal diseases during COVID-19: we should be prepared. J De Mycol Med 30:100971

15. Arastehfar A, Carvalho A, Van de Veerdonk FL, Jenks JD, Koehler P, Krause R, Cornely OA, Perlin DS, Lass-Florl C, Hoenigl M (2020) COVID-19 associated pulmonary aspergillosis (CAPA)- from immunology to treatment. J Fungi 6(2):91

16. Alanio A, Delliere S, Fodil S, Bretagne S, Megarbane B (2020) High prevalence of putative invasive pulmonary aspergillosis in critically ill COVID-19 patients. MedRxiv

17. Blaize M, Mayaux J, Nabet C, Lampros A, Marcelin AG, Thellier M, Piarroux R, Demoule A, Fekkar A (2020) Fatal invasive aspergillosis and coronavirus disease in an immunocompetent patient. Emerg Infect Dis 26(7):1636

18. Koehler P, Cornely OA, Bottiger BW, Dusse F, Eichenauer DA, Fuchs F, Hallek M, Jung N, Klein F, Persigehl T, Rybniker J (2020) COVID-19 associated pulmonary aspergillosis. Mycoses 63(6):528-534

19. Bellanger AP, Persat F, Foulet F, Bonnal C, Accoceberry I, Angebault C, Angoulvant A, Augereau O, Bailly E, Bert F, Bonhomme J (2020) Antifungal susceptibility testing practices in mycology laboratories in France, 2018. J de Mycol Med $8: 100970$

Publisher's Note Springer Nature remains neutral with regard to jurisdictional claims in published maps and institutional affiliations. 